\title{
Ab initio study of exciton transfer dynamics from a core-shell semiconductor quantum dot to a porphyrin-sensitizer
}

\author{
Dmitri S. Kilin ${ }^{\text {a }}$, Kiril Tsemekhman ${ }^{\text {a }}$, Oleg V. Prezhdo a,*, \\ Eduard I. Zenkevich ${ }^{\mathrm{b}}$, Christian von Borczyskowski ${ }^{\mathrm{c}}$

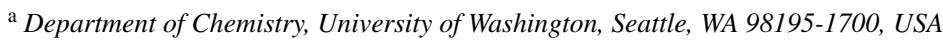 \\ ${ }^{\mathrm{b}}$ Institute of Molecular and Atomic Physics, National Academy of Science of Belarus, F. Skaryna Ave. 70, 220072 Minsk, Belarus \\ ${ }^{\mathrm{c}}$ Center for Nanostructured Materials and Analytics, Institute of Physics, Chemnitz University of Technology, \\ D-09107 Chemnitz, Germany
}

Received 25 October 2006; received in revised form 15 February 2007; accepted 19 February 2007 Available online 23 February 2007

\begin{abstract}
The observed resonance energy transfer in nanoassemblies of CdSe/ZnS quantum dots and pyridyl-substituted free-base porphyrin molecules [Zenkevich et al., J. Phys. Chem. B 109 (2005) 8679] is studied computationally by ab initio electronic structure and quantum dynamics approaches. The system harvests light in a broad energy range and can transfer the excitation from the dot through the porphyrin to oxygen, generating singlet oxygen for medical applications. The geometric structure, electronic energies, and transition dipole moments are derived by density functional theory and are utilized for calculating the Förster coupling between the excitons residing on the quantum dot and the porphyrin. The direction and rate of the irreversible exciton transfer is determined by the initial photoexcitation of the dot, the dot-porphyrin coupling and the interaction to the electronic subsystem with the vibrational environment. The simulated electronic structure and dynamics are in good agreement with the experimental data and provide real-time atomistic details of the energy transfer mechanism.
\end{abstract}

(C) 2007 Elsevier B.V. All rights reserved.

Keywords: Singlet oxygen; Photocytotoxicity; Cancer therapy; Exciton transfer; Core-shell semiconductor quantum dot; Porphyrin photosensitizer

\section{Introduction}

Recent advances in the synthesis of nanostructures with controllable size, shape, and optical properties make them desirable materials for new technologies including spintronics [1], quantum computing [2,3] and photovoltaics [4-10]. Quantum dots (QDs) form the foundation of many novel devices such as thermopower machines [11], field-effect transistors [12], lightemitting diodes [13], lasers [14], and quantum emitter antennas [15]. Functionalizing inorganic QDs with organic ligands prepares them for applications in various biological and medical fields [16], including in vivo fluorescent biological imaging [17] and cytotoxicity [18]. Organically sensitized QDs are used in drug design. In particular, assemblies of QDs with photosensiting dyes maximize production of singlet oxygen, thereby

\footnotetext{
* Corresponding author. Tel.: +1 2062213931.

E-mail address: prezhdo@u.washington.edu (O.V. Prezhdo).
}

providing a novel approach to photo-dynamic therapy (PDT) $[19,20]$.

PDT is a highly promising application of nanotechnology to the selective destruction of malignant tissues, such as cancer cells. It is a rapidly developing field [21] that can greatly benefit from support by theory and simulation. Realization of PDT relies on singlet oxygen, which is generally accepted as the main mediator of photocytotoxicity in PDT and causes oxidation and degradation of membranes of malignant cells [22,23]. Since a direct excitation from the triplet ground state $\left({ }^{3} \mathrm{O}_{2}\right)$ to the singlet excited state $\left({ }^{1} \mathrm{O}_{2}\right)$ is forbidden by spin selection rules, oxygen is activated with a sequence of energy transfer events involving a photosensitizer [24]. The ${ }^{1} \mathrm{O}_{2}$ agent can be effectively generated using self-assembled aggregates of tetrapyrrole dyes and small $(3-10 \mathrm{~nm})$ QDs covered by ligands [25]. The commonly used tetrapyrrole dyes include phtalocyanin [26] and porphyrin [27-30]. QDs are used for the following reasons: (i) they store excitation energy and distribute it to many dye molecules, which reside on the QD surface and produce singlet oxygen; (ii) QDs 
are efficient photon harvesters, since their absorption spectrum is broader than the spectra of typical organic dyes used in PDT; and (iii) the photon absorption of QDs can be tuned to the spectral transparency window of human skin. Although metallic QDs can be less toxic [31], semiconductor QDs have important advantages for medical applications. Semiconductor QDs are more easily sensitized with organic molecules and polymers, which reduce QD toxicity and are required in the applications. Compared to their metallic counterparts that show photoinduced activity in the same spectral region, semiconductor QDs are substantially smaller in size, and are therefore are much more easily delivered to biological tissues.

The generation of singlet oxygen with the dye-QD assemblies involves two steps. First, the photoexcitation moves from the QD to the dye. Second, the dye transfers the energy to the oxygen present in the surrounding solvent. Clear understanding of the energy transfer mechanisms and pathways involved in this two-step process is essential to the design of effective PDT drugs. The pathway taken by the photoexcitation in the QD-dye nanoassembly is best described with the modern theory of energy transfer, which is greatly motivated by search for novel energy sources, design of artificial light-harvesting systems, and understanding the efficient light harvesting seen in nature [32-38]. The energy and electron transfer processes in the light-harvesting systems are driven by the ultrafast photoinduced evolution of the electronic degrees of freedom, which are strongly affected by the dynamic reorganization of the quantized vibrational modes [39-41]. Therefore, theoretical description of the transfer processes requires explicit modeling of the coupled electronic and vibrational dynamics together with bath-induced dephasing and renormalization of the system energies [40-45].

The long-range excitation transfer between chromophores in molecular aggregates is well described by the Förster theory [46], in which the transfer rate is proportional to the overlap of the donor emission and acceptor absorption spectra. Among many applications, the theory successively describes the enhancement of absorption efficiency in the networks of chromophores acting as solar radiation antennas [32-35]. The theory assumes that the electrons undergoing excitation transfer are in contact with a thermal phonon bath, which can both rapidly accomodate excess electron energy and provide thermal energy to the electrons.

Explicit dynamics are particularly important for those degrees of freedom that are not thermalized on the timescale of the transfer [47]. A number of quantum and semiclassical approaches have been developed for the description of transfer dynamics in large systems [48-54]. Mixed quantum-classical molecular dynamics, in which a few quantum mechanical electronic or vibrational modes are coupled to many explicit classical degrees of freedom, are used for modeling of transfer reactions, which produce substantial differences in the electrostatic interactions in the donor and acceptor configurations and generate large solvation and reorganization energies [55-61].

An intermediate description between the rate theories and molecular dynamics is provided by the reduced density matrix methods [38,41-43,62-64], which couple several explicit electronic or vibrational modes to a thermal bath of many modes.
The reduced density matrix approach is particularly suitable for the present study, which is aimed at modeling the time-resolved experimental data on the evolution of the electronic system coupled to the phonon environment. Since the excitation transfer in the dot-dye assembly causes no bond breaking or other major chemical events, the nuclear motions change little and can be treated in the harmonic approximation. The phonon motions are orders of magnitude faster than the excitation transfer and remain thermalized during the course of the transfer. In certain limits, the master equations derived for the reduced density matrices may be cast into the form of quantum jump equations [51,65-67] that deal with individual trajectories rather than ensembles. These single molecule simulations can provide additional insights into the course of a photoreaction. Since the experiments of current interest [28] report ensemble averaged data, we use the reduced density matrix description.

The paper presents an ab initio model for the photoinduced energy transfer in the colloidal core-shell $\mathrm{CdSe} / \mathrm{ZnSe}$ wurtzite $\mathrm{QD}$, whose surface is sensitized with a meta-dipyridyl-porphyrin dye [68]. The ab initio model is used to investigate the energy transfer dynamics in real-time. The electronic structure calculations are performed using density functional theory (DFT) and explicitly include the CdSe core, the $\mathrm{ZnSe}$ shell and the porphyrin sensitizer. The density matrix model is designed to represent the dipole-dipole Förster coupling mechanism and to account for the electron-phonon interactions. The simulation explicitly describes transfer, relaxation, and dephasing of the two-particle excitations on a nanosecond timescale. The next section describes the details of the $a b$ initio calculations of the electronic structure and optical properties of the dye-dot assembly and introduces the reduced density matrix description of the excitation transfer. Following the theory section, we discuss the simulation results and conclude with a summary and an outline of future research directions.

\section{Theory and simulation details}

In this section, we describe QD-dye system used in the simulations (Fig. 1), characterize its atomic structure and report the details of the $a b$ initio electronic structure and quantum dynamics simulations.

\subsection{Atomic structure of the $Q D-$ porphyrin composite}

Inorganic QDs attract fundamental interest because of their long-lived electronic excitations and high fluorescence yields, which are made possible by weaker electron-phonon interactions compared to organic systems and by quantum confinement relative to the bulk. Quantization of the electronic energy levels due to finite size of the dots results in the phonon bottleneck [4], which inhibits efficient non-radiative relaxation. In order to take advantage of the unique electronic and optical properties of QD, the unsaturated coordination sites on the surface should be removed. The dangling bonds can be saturated by several mechanisms, including (i) surface self-healing, (ii) surface ligation, and (iii) QD capping by a shell of another inorganic semiconductor material with a similar geometric structure and a 


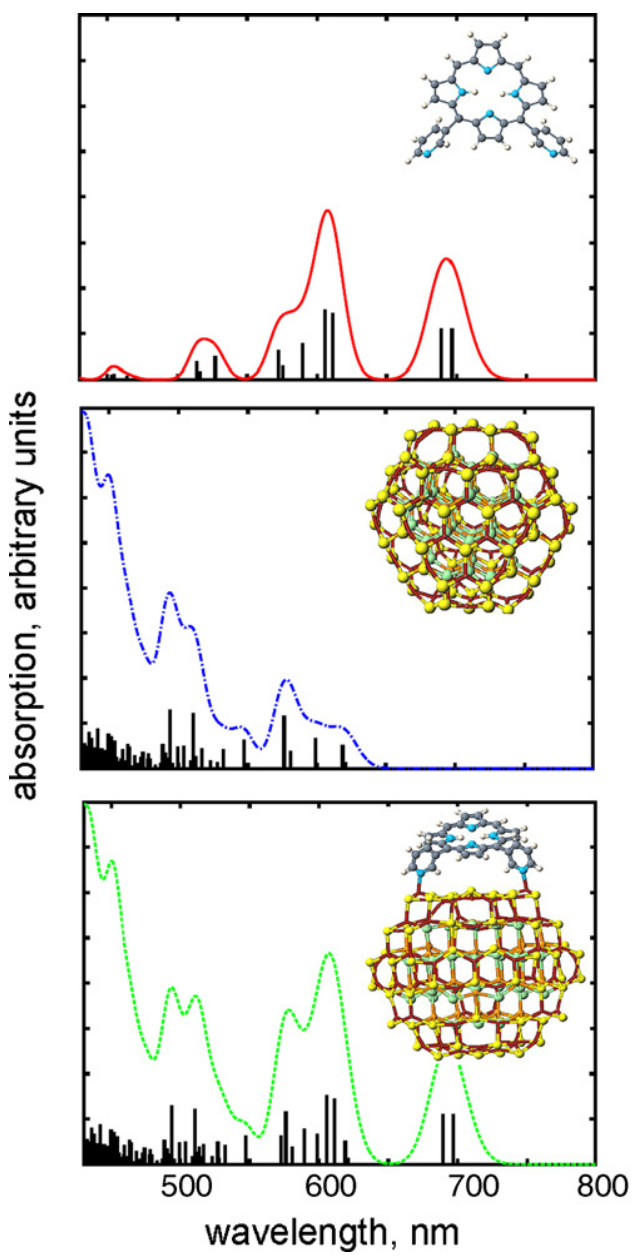

Fig. 1. Calculated absorption spectrum and molecular structure of the dipyridyl porphyrin (top panel), the core-shell CdSe/ZnS quantum dot (middle panel) and the dot-dye composite (bottom panel).

larger band-gap. The unsaturated dangling bonds generate surface states with energies inside the band-gap. The surface states create excitation traps, provide new relaxation pathways, and generally, deteriorate the optical properties of the dot. Saturation of the dangling bonds removes the surface states and opens up the band-gap. Surface self-healing and reconstruction facilitates formation of additional bonds between the surface atoms. Surface ligands compensate dangling bonds and, at the same time, preserve the ideal crystal structure of the dot. QD capping with a shell of another semiconductor with a larger band-gap greatly improves the optical properties of the dot. Compared to the highly mobile atoms of a self-healed surface or flexible surface ligands, both of which create strong electron-phonon coupling, a rigid semiconductor shell slows down radiationless relaxation and enhances fluorescence. Such core-shell QDs have been used in the PDT experiments of interest [28] and form the focus of our study.

Mass-spectroscopy experiments indicate [69] that structures and properties of smaller QDs are better defined than those of larger dots. Smaller QD clusters contain fixed numbers of atoms and form only few stable configurations that both maintain the original crystal structure and minimize surface energy. The QD considered here comprises 222 atoms total, including 66 atoms in the $\mathrm{Cd}_{33} \mathrm{Se}_{33}$ core and 156 atoms in the $\mathrm{Zn}_{78} \mathrm{~S}_{78}$ shell. Both the core and the shell maintain the wurtzite structure of bulk $\mathrm{CdSe}$ and $\mathrm{ZnS}$ upon geometry optimization, middle panel of Fig. 1.

The free-base porphyrin is a tetrapyrrole organic dye that shows a high optical absorption yield and is an essential part of many natural and artificial light-harvesting complexes. Due to its lone electron pairs localized in the nitrogen atoms, the dipyridyl-substituted porphyrin shown in the top panel of Fig. 1 can easily self-assemble with the QD. The nitrogen atoms of the bridging pyridyl groups strongly bind to $\mathrm{Zn}$ surface atoms separated by a matching distance. The orbitals of the porphyrin that are responsible for its optical properties are affected by neither the dipyridyl substituents nor the surface binding.

The dye-dot assembly used in the current study, bottom panel of Fig. 1, is a simplified version of the experimentally studied system $[28,29]$. It is designed to capture the key properties of the real system at the fully atomistic level, while allowing the $a b$ initio description of its electronic structure. The simulated QD is about half the size of the dots used in the experiments. Both the CdSe core and the $\mathrm{ZnS}$ shell are essential for the description of the quantum dot electronic structure and are included. The TOPO-ligands that cover the outer $\mathrm{ZnS}$ layer of the dot in addition to the porphyrin dyes are excluded. The ligands do not influence the electronic properties of the dot in the experimentally relevant energy range, since the $\mathrm{CdSe}$ dangling bonds are saturated by the $\mathrm{ZnS}$ shell. The surface ligands should have little effect on the dye-dot donor-acceptor coupling as well, since the dye is directly bound to the dot.

\subsection{Electronic structure}

The electronic structure of the core-shell QD sensitized with the porphyrin dye is investigated by $a b$ initio density functional theory (DFT). Among other advantages, DFT works well with transition elements containing d-electrons and is scalable to the system size required for the present study. The DFT simulations were performed with the VASP code that is particularly efficient with semiconductor and metallic systems [70-72]. The core electrons were simulated using the Vanderbilt pseudopotentials [73], while the valence electrons were treated explicitly. The generalized gradient functional due to Perdew and Wang [74] was used to account for the electron exchange and correlation effects. The simulations were performed using a basis of $9 \times 10^{5}$ plane waves corresponding to the energy cutoff of $220 \mathrm{eV}$. The electronic structure was converged to the $0.001 \mathrm{eV}$ tolerance limit. In order to use efficient fast Fourier transforms, VASP employs periodic boundary conditions. A vacuum layer of at least $7 \AA$ was added between the images to avoid spurious interactions between the periodic images of the system.

The geometry of the combined system was first fully optimized using molecular mechanics within the HyperChem software package [75]. The geometry was further optimized with VASP. The ab initio DFT optimization was essential for treating the spatial anisotropy of the forces produced by the $\mathrm{Zn}$ and $\mathrm{N}$ atoms that form the dye-dot coordination bond. While 
van der Waal's interactions are known to challenge conventional DFT, requiring special DFT approaches [76,77], the coordination binding of the dye to the dot is analogous to hydrogen bonding $[59,78]$ which is stronger than van der Waal's interactions, and is well represented by standard DFT. Since all dot and dye atoms were allowed to move, the DFT geometry optimization accounted for the intra-dye relaxation induced by the semiconductor surface, the changes in the surface induced by the interaction with the dye, and the optimization of the binding geometry.

The optical properties of the dye-dot composite are described within the Kohn-Sham orbital picture. The orbital representation is well justified for the semiconductor QD, since its optical properties are dominated by quantum confinement [4]. The kinetic energy of quantum confinement is much greater than the Coulomb energy of electrostatic attraction between the electron and hole occupying the orbitals involved in the photoexcitation. While more advanced approaches, such as the Bethe-Salpeter theory [79] or DFT including exact exchange terms [80], can produce better agreement with the experimental data, they are much more computationally demanding and cannot be applied yet to a system of this size.

It should be noted that due to the presence of the heavy elements, the spin-orbit interaction (SOI) plays an important role in the optical properties of QDs by mixing singlet and triplet states [81]. The strength and importance of SOI is system and size dependent. For instance, $\mathrm{SOI}$ is less prominent in $\mathrm{PbSe} \mathrm{QDs}$, in which the exciton radius is large, around $46 \mathrm{~nm}$, and quantum confinement effects dominate [82]. The exciton radius in $\mathrm{CdSe}$ is smaller. SOI in CdSe QDs suppresses the singlet character of the lowest energy exciton to as low as 30\% [81]. Similarly, the relatively closely spaced singlet and triplet states of the porphyrin molecule are noticeably coupled by SOI, which creates the conditions required for creation of singlet oxygen. Because of the mixed spin character of the exciton, the exciton transfer studied in present work effectively couples both singlet and triplet states $[83,84]$. The transfer dynamics is analyzed here without detailing the total angular momentum of the exciton and with explicit focus on the time-domain.

Both the photoexcitation intensity and the donor-acceptor coupling in the Förster theory [46] depend on the transition dipole moments between the electron and hole states. Within the plane-wave DFT formalism, the Kohn-Sham wavefunctions are given in the momentum representation $\left|\psi_{\nu, \mathbf{G}}\right\rangle$. Using the equivalence between the coordinate and momentum representation, the transition dipole moments are calculated according to the formula $\mathbf{d}_{\mu \nu}=\sum_{|\mathbf{G}|<\mathbf{G}_{\text {cutoff }}} \psi_{\mu, \mathbf{G}}^{*}|\mathbf{G}| \psi_{\nu, \mathbf{G}}$, where $\mu$ and $v$ denote the electron and hole states, respectively, $\mathbf{G}$ are the reciprocal lattice vectors, and $\mathbf{G}_{\text {cutoff }}$ is the value of the reciprocal lattice vector where the Fourier expansion is truncated. The density of electronic states is calculated by assigning a Gaussian profile with the natural linewidth to each excited state: $n(\epsilon)=\sum_{\mu}(1 / \sqrt{2 \pi \sigma}) \exp \left\{-\left(\left(\epsilon-\epsilon_{\mu}\right)^{2} / 2 \sigma\right)\right\}$. The system absorption spectrum $A(\epsilon)$ is calculated as a superposition of individual peaks, whose amplitudes are determined by the oscillator strength: $A(\epsilon)=\sum_{\mu \nu} \epsilon\left|\mathbf{d}_{\mu \nu}\right|^{2} P_{\nu}(1-$
$\left.P_{\mu}\right)(1 / \sqrt{2 \pi \sigma}) \exp \left\{-\left(\left(\epsilon-\left|\epsilon_{\mu}-\epsilon_{\nu}\right|\right)^{2} / 2 \sigma\right)\right\}$. Here $P_{\mu}, P_{\nu}$ are the populations of orbitals $\mu, v$. Since the dot and the dye are well separated across the interface, the states are localized on either the dot or the dye.

\subsection{Excitation dynamics}

The dynamics of the energy transfer between the CdSe/ZnS crystal and the porphyrin dye is described by the reduced density matrix formalism that allows us to focus on the exciton subsystem, while incorporating the coupling to the phonon bath. Below, $\mu, v$ label orbitals, $i=\{\mu, v\}$ label excitons, and $A=\{i, j\}$ label pairs of excitons. The equation of motion for the exciton density matrix is

$\frac{\partial}{\partial t} \rho=i \hbar \underbrace{[H, \rho]}_{\text {elastic }}-i \hbar^{2} \underbrace{\left\langle\left[H^{\mathrm{int}},\left[H^{\mathrm{int}}, \rho\right]\right]\right\rangle}_{\text {inelastic }}$.

The first term in the above equation is responsible for elastic, Hamiltonian, reversible dynamics. The second term represents inelastic, irreversible evolution appearing due to interaction with phonons. The diagonal part of the elastic term involves the difference between the electron and hole orbital energies

$H_{i i}=H_{\mu \nu, \mu \nu}=\epsilon_{\mu}^{\text {electron }}-\epsilon_{\nu}^{\text {hole }}$

for the pairs of orbitals that are both localized on either the dot or the dye. The dipole-dipole interaction defines the off-diagonal part of the elastic term and describes the exciton-exciton coupling by the Förster mechanism:

$H_{i, j}=H_{\mu v, \kappa \lambda}=\frac{\mathbf{d}_{i}^{\mathrm{dye}} \cdot \mathbf{d}_{j}^{\mathrm{dot}}}{R_{i j}^{3}}-\frac{3\left(\mathbf{d}_{i}^{\mathrm{dye}} \cdot \mathbf{R}_{i j}\right)\left(\mathbf{d}_{j}^{\mathrm{dot}} \cdot \mathbf{R}_{i j}\right)}{R_{i j}^{5}}$,

where

$\mathbf{d}_{i}^{\text {dye }}=\mathbf{d}_{\mu \nu}^{\text {dye }}=e \mathbf{r}_{\mu \nu}^{\text {dye }}=e\left\langle\psi_{\mu}^{e, \text { dye }}|\mathbf{r}| \psi_{\nu}^{h, \text { dye }}\right\rangle$,

$\mathbf{d}_{j}^{\mathrm{dot}}=\mathbf{d}_{\kappa \lambda}^{\mathrm{dot}}=e \mathbf{r}_{\kappa \lambda}^{\mathrm{dot}}=e\left\langle\psi_{\kappa}^{e, \operatorname{dot}}|\mathbf{r}| \psi_{\lambda}^{h, \mathrm{dot}}\right\rangle$.

The indices $\mu, v$ refer to the electron and hole acceptor orbitals localized on the dye, while the indices $\kappa, \lambda$ describe the electron and hole donor orbitals localized on the QD. The vector $\mathbf{R}_{i j}$ connecting the transition dipole moments of the dye and QD is defined by

$\mathbf{R}_{i j}=\mathbf{r}_{i}^{\text {dye }}-\mathbf{r}_{j}^{\text {dot }}, \quad \mathbf{r}_{i}^{\text {dye }}=\frac{\mathbf{r}_{\mu}+\mathbf{r}_{v}}{2}, \quad \mathbf{r}_{j}^{\text {dot }}=\frac{\mathbf{r}_{\kappa}+\mathbf{r}_{\lambda}}{2}$,

where

$\mathbf{r}_{\mu}=\left\langle\psi_{\mu}^{e, \text { dye }}|\mathbf{r}| \psi_{\mu}^{e, \text { dye }}\right\rangle$, etc.

The vectors $\mathbf{r}_{i}^{\text {dye }}$ and $\mathbf{r}_{j}^{\text {dot }}$ defining the centers of mass (COM) of each exciton are computed by averaging the COM of electron and hole forming the exciton. The scalar $R_{i j}$ is the length of the vector $\mathbf{R}_{i j}$. The excitation transfer is dominated by the pairs of dot and dye states that have the largest transition dipole moments. Typical values of the transition dipole moments, exciton transfer distances, and dipole-dipole coupling matrix elements are $2.5 \mathrm{D}$, $15 \AA$, and $5 \mathrm{meV}$, respectively. 
The irreversible evolution is constructed using the electron-phonon interaction Hamiltonian that is approximated by a bilinear product of the transition operator and the nuclear coordinate operator $\mathbf{Q}_{a}$.

$H_{i j}^{\mathrm{int}}=H_{\mu \nu, \kappa \lambda}^{\mathrm{int}}=\sum_{a} \mathbf{D}_{\mu v, \kappa \lambda} \mathbf{Q}_{a}$,

$\mathbf{D}_{\mu \nu, \kappa \lambda}=\underbrace{\mathbf{d}_{\mu \kappa}}_{\text {electron goes }} \underbrace{\delta_{v \lambda}}_{\text {hole stays }}+\underbrace{\delta_{\mu \kappa}}_{\text {electron stays }} \underbrace{\mathbf{d}_{v \lambda}}_{\text {hole goes }}$.

The assumed bilinear form describes transition of either electron or hole as symbolized by the first and second contributions to D.

In order to study the dynamics on the experimentally relevant nanosecond timescale, we avoid calculating the long-time molecular dynamics of the dot-dye system in an explicit form. Instead, we determine the frequencies of the system phonon modes that couple to the electronic states by Fourier transforming the relevant electronic state energy gaps obtained from a short, picosecond molecular dynamics run, and construct the bath spectral density. Averaging over the vibrations leads to the following equation of motion for the reduced density matrix of the exciton

$\frac{\partial}{\partial t} \rho_{i j}=\sum_{k l}\left(\frac{i}{\hbar} L_{i j k l}+R_{i j k l}\right) \rho_{k l}$

$L_{i j k l}=H_{i k} \delta_{l j}-\delta_{i k} H_{l j}$,

$R_{i l k j}=\delta_{i l}\left(-\sum_{m} p_{i m} \delta_{i k} \delta_{j i}+p_{j i} \delta_{j k}\right)-\delta_{i k} \delta_{j l} \gamma_{k j}$.

The equation contains the Liouville $L_{i j k l}$ and Redfield $R_{i j k l}$ superoperators. The Redfield tensor is constructed based on the dephasing rate $\gamma_{i j}=(1 / 2) \sum_{k}\left(p_{i k}+p_{k j}\right)$, the transition probabilities $p_{i j}=\pi K_{i j}^{2} \mathbf{D}_{i j}^{2} J\left(\omega_{i j}\right)$, the square of the transition operator $\mathbf{D}_{i j}$, the dielectric constant of the medium $\epsilon$ that defines $K_{i j} \simeq 1 / \epsilon$, and the spectral density $J(\omega)=\rho(\omega) n(-\omega)$. The latter involves the density of phonon modes $\rho(\omega)$, which is obtained from the picosecond trajectory, and the thermal boson distribution $n(\omega)=\left[\exp \left(\hbar \omega / k_{\mathrm{B}} T\right)-1\right]^{-1}$ [64]. The equation is solved by numeric diagonalization of the super-operator $(L+R) \rho_{\Xi}=\Omega_{\Xi} \rho_{\Xi}$, providing the exciton density matrix, the excited state populations, and the fluorescence spectrum

$\rho(t)=\sum_{\Xi}\left\langle\rho(0) \mid \rho_{\Xi}\right\rangle \exp \left\{-i \Omega_{\Xi} t\right\}$

$P_{\mu \nu}(t)=\rho_{\mu \nu, \mu \nu}(t)$,

$F(\epsilon, t)=\sum_{\mu \nu} \epsilon \mathbf{d}_{\mu \nu}^{2} P_{\mu \nu}(t) \frac{1}{\sqrt{2 \pi \sigma}} \exp \left\{-\frac{\left(\epsilon-\left[\epsilon_{\mu}-\epsilon_{\nu}\right]\right)^{2}}{2 \sigma}\right\}$,

respectively, at any instant of time. The initial excitation involves all energetically accessible exciton states localized on the dot, representing excitation by a short optical pulse covering a broad energy range, as expected in PDT treatments.

\section{Results and discussion}

The reported simulations provide an atomistic ab initio description of the electronic structure of the dot-dye assembly and uncover important features of the exciton transfer dynamics, as detailed below.

\subsection{Electronic structure}

The shape and structure of the calculated absorption lines, Fig. 1, are similar to the lowest excitations observed in the experiment: [28] the two pairs of lines are separated by about $100 \mathrm{~nm}$ and have different intensities. The calculated lowest energy pair of lines occurs at $v_{\text {calc }}=689,697 \mathrm{~nm}$, which is slightly larger than corresponding experimental value, $v_{\exp } \simeq 650 \mathrm{~nm}$, see Table 1 . The calculated second pair of transitions $v_{\text {calc }}=606$, $611 \mathrm{~nm}$ also has a longer wavelength than measured transitions, $v_{\text {exp }} \simeq 550 \mathrm{~nm}$. Table 1 presents the excitation energies obtained using the Kohn-Sham orbital energy differences, as well as the constrained DFT calculations, in which the total energies of the ground and excited states are computed by constraining the electrons to occupy the selected orbitals. The constrained DFT results are in excellent agreement with the experimental data, even though they have been obtained with semi-local DFT, which is known for its systematical errors in long-range electron transfer $[85,86]$. The calculation excludes the vibronic progression of $0-0,0-1$, etc. excitations, involving the first and higher vibrational levels associated with the excited electronic state. Since the vibronic progression moves the excitation maximum to higher energies, it should bring the theory into closer agreement with the experiment. The density of states of the dot-dye composite is approximately equal to the sum of the densities of states of the isolated subsystems. The absorption spectrum of the composite is also approximately equal to the sum of the dot and dye absorption spectra (Fig. 1).

The QD and porphyrin orbitals that are involved in the lowest energy excitations are shown in Fig. 2. The lowest unoccupied molecular orbital (LUMO) contributing to the dot exciton is localized primarily in the core region of the dot. Only small portions of the electron density extend onto the QD surface and the dye. The highest occupied molecular orbital (HOMO) is localized away from the dye and shows significant contributions from the shell region. The second, third and fourth excitations localized on the dot promote the electron from different occupied orbitals into the same unoccupied orbital as the first excitation. This is because the spacing of the energy levels is smaller

Table 1

Experimental [28] and calculated wavelengths of the lowest optical transitions in the free-base porphyrin

\begin{tabular}{llll}
\hline Orbital pair & $\begin{array}{l}\text { Orbital energy } \\
\text { gap }\end{array}$ & $\begin{array}{l}\text { Constrained } \\
\text { DFT }\end{array}$ & Experiment \\
\hline HOMO/LUMO & 697 & 663 & 650 \\
HOMO/LUMO + 1 & 689 & 649 & 649 \\
HOMO - 1/LUMO & 611 & 574 & 550 \\
HOMO - 1/LUMO + 1 & 606 & 564 & 550 \\
\hline
\end{tabular}




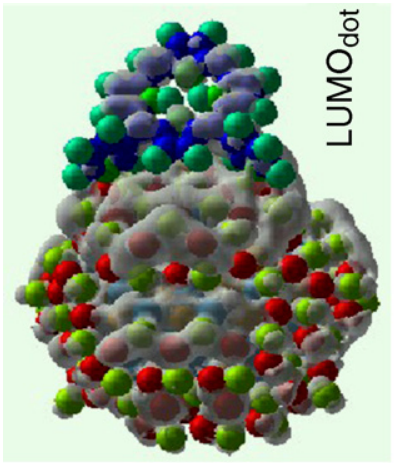

$\lambda=621 \mathrm{~nm}$
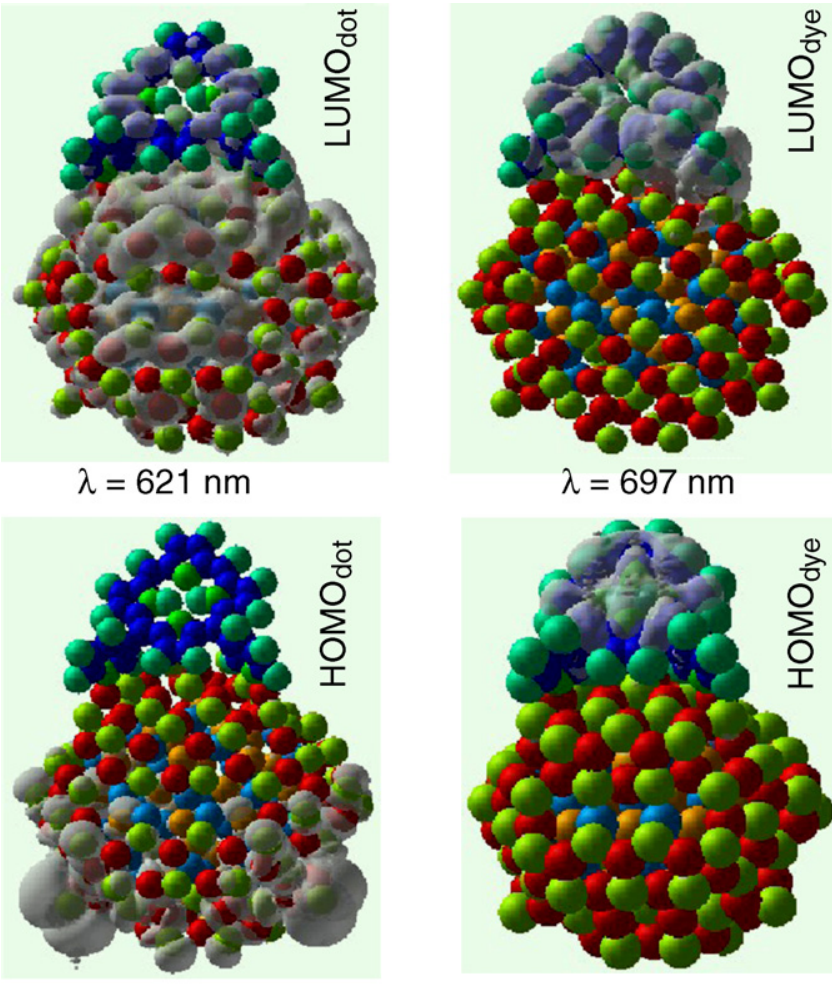

color code: $\mathrm{H} \mathrm{C} \mathrm{N} \mathrm{Cd} \mathrm{Se} \mathrm{Zn}$ S

Fig. 2. Densities of HOMO and LUMO localized on the QD (left panel) and the porphyrin dye (right panel).

for the occupied orbitals than for the vacant orbitals, as rationalized by the significantly larger effective mass of the hole compared to that of the electron in $\mathrm{CdSe}$, and in most semiconductor QDs in general $[4,87]$. The orbitals that generate the lowest energy exciton residing on the dye are well isolated and have little density on the dot. The HOMO is entirely on the
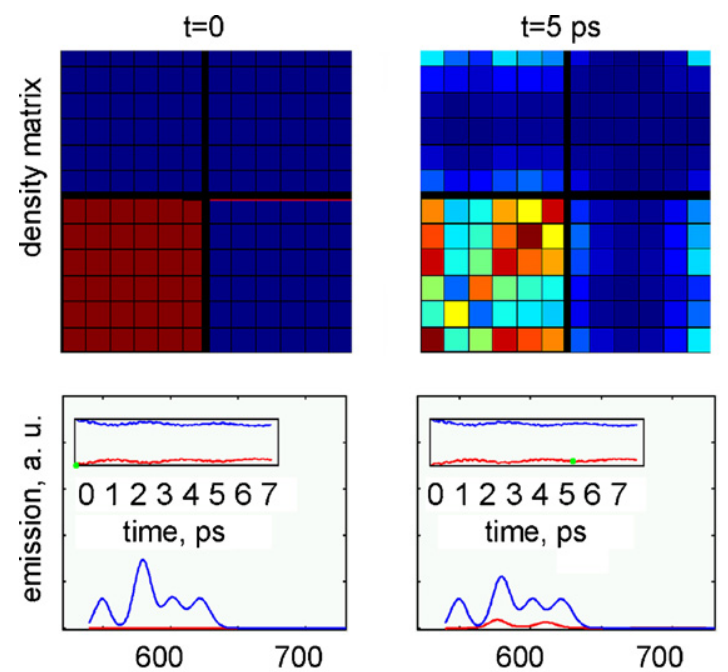

porphyrin, while the LUMO shows some dye-dot mixing. The $\mathrm{HOMO}$ and $\mathrm{HOMO}+1$ of the porphyrin are degenerate, explaining why the first and second excitations of the porphyrin are of nearly the same energy. These two transitions belong to the Qband [88]. The orbital densities shown in Fig. 2 indicate that the dye-dot coupling occurs more efficiently through the vacant orbitals occupied by the excited electron, rather than through the occupied orbitals supporting the hole. Since several QD excitons share the same vacant orbital, the dot-dye coupling as well as the rate and efficiency of the exciton transfer should exhibit little energy dependence.

The relative energies of the band edges of the QD and of the HOMO-LUMO of the dye determine the direction of the exciton transfer. The transfer occurs by the Förster mechanism with the rate which is directly proportional to the overlap $J \simeq \int d \epsilon \epsilon^{-4} F_{\mathrm{D}}(\epsilon) A_{\mathrm{A}}(\epsilon)$ of the emission spectrum of the donor species $F_{\mathrm{D}}$ and the absorption spectrum of the acceptor species $A_{\mathrm{A}}$. The lowest excitation energy band of the dot lies around $600 \mathrm{~nm}$ and overlaps with the second band of the dye (Fig. 1). This overlap allows resonance transfer of the exciton from the dot to the dye. Once on the dye, the exciton relaxes to the dye lowest energy band around $700 \mathrm{~nm}$. The $700 \mathrm{~nm}$ dye band has no counterpart in the $\mathrm{QD}$, such that the relaxed exciton cannot move back from the dye onto the dot. The computed energies and overlaps of the spectral bands of the QD and the porphyrin dye rationalize the observed directionality of the exciton transfer in the dot-dye composite.

\subsection{Exciton dynamics}

The evolution of the density matrix of the dot-dye system is shown in the upper part of Fig. 3. The corresponding evolution of the emission spectra of the dot and the dye species is given in the lower part of the figure. At the initial time both diagonal and off-diagonal elements of the dot-exciton block of the density
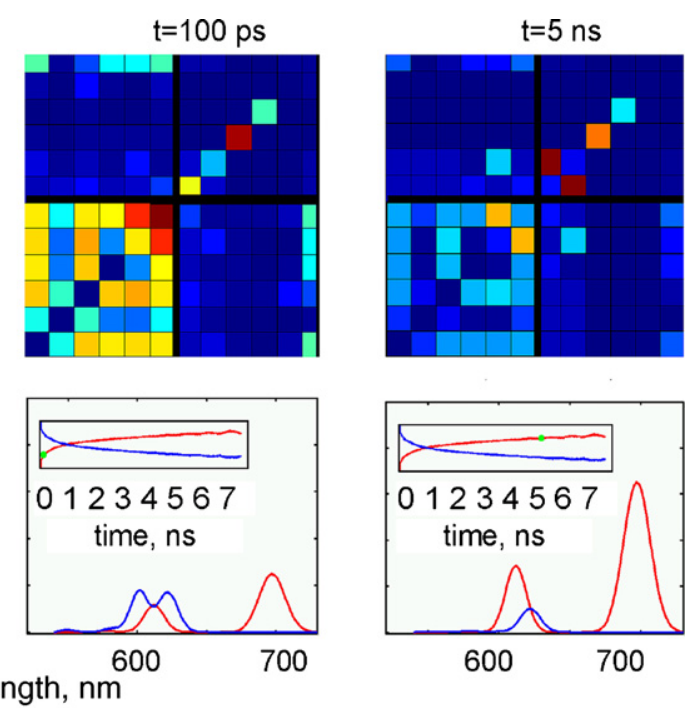

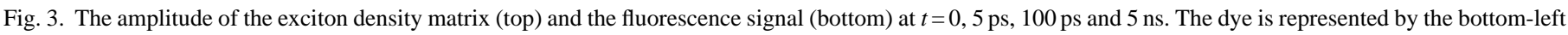

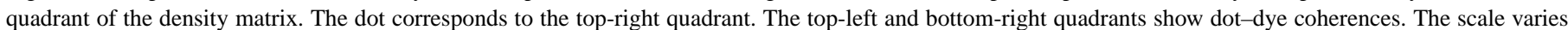

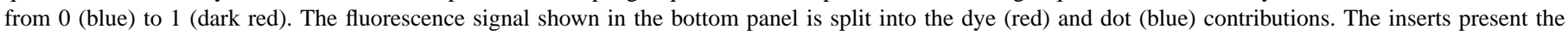
time-dependent localizations of the exciton of the dot and the dye, with the green circle showing the current moment in time. 
matrix are non-zero, simulating a QD-dye system driven by a short laser pulse into a superposition of several excited states. These excited states are revealed in the emission peaks seen at $550,580,600$, and $630 \mathrm{~nm}$, and are attributed to the dot. The offdiagonal elements of the dot-exciton block of the density matrix decay on a hundred femtosecond to a few picosecond timescale, caused by the relatively fast dephasing of the electronic degrees of freedom due to coupling to multiple vibrational modes. The occupations of the diagonal matrix elements of the dot-exciton block move towards the lowest energy excitation. This energy relaxation is driven by the phonon bath as well and is manifest in the decrease of the emission intensity of the 550 and $580 \mathrm{~nm}$ dot bands. The relaxation is significantly slower than dephasing, since the energy exchange involved in the relaxation depends on the availability of vibrational modes that are resonant with the energy gaps between the electronic states. On the other hand, dephasing does not require energy exchange and is induced by phonon-induced fluctuations of the electronic energy levels.

Approximately on the same timescale as the thermal relaxation inside the dot, non-zero matrix elements appear in the blocks, describing coherence between the dot and dye states. The coherence transfer almost immediately leads to the population transfer seen in the rise of the diagonal elements of the dye block. The build-up of the population in the dye states results in the emission at 590 and $620 \mathrm{~nm}$ attributed to the dye. Over time, the dye emission shifts towards $700 \mathrm{~nm}$. The $610 \mathrm{~nm}$ dye band continues to emit for a long time, indicating that the electronvibrational relaxation is relatively slow within the dye compared to the intra-dot relaxation, as should be expected based on the significantly larger spacing between the dye energy levels. Per- sistent coherence observed at the final steps of the dynamics are attributed to the dye exciton degeneracies.

Next, we consider in more detail the dynamics that occurs on the shorter and longer timescales, during the first $10 \mathrm{ps}$ and $10 \mathrm{~ns}$, respectively. While the sub-picosecond dynamics is also accessible in our simulation, the details of such dynamics associated with deviations from the thermal rate can be reliably understood only with explicit treatment of molecular vibrations. The time-resolved fluorescence signal of the dot-dye aggregate is presented in Fig. 4, upper panel. The signal is calculated as a superposition of the bands whose intensities are proportional to the population of the relevant excitonic state times the appropriate oscillator strength. The linewidth of each band corresponds to room temperature. The lines around 550 and $580 \mathrm{~nm}$ correspond to the dot states. The band in the range of $620-600 \mathrm{~nm}$ contains both dot and dye emission. The line around $700 \mathrm{~nm}$ is due to the dye alone. The oscillations in the signal around 580 and $700 \mathrm{~nm}$ with the period of around $2 \mathrm{ps}$ indicate a relatively large coupling between the dot and dye states.

The calculations produce a general physical picture of the energy transfer in the aggregate. The initially populated dot states emit at 550,580,620 nm. Over time, thermalization through phonons induces relaxation from the higher to the lower energy dot states, which is reflected in the shift of the emission signal occurring at around $30 \mathrm{ps}$. The relaxation is followed by resonance energy transfer between the dot and the dye states in the $600-620 \mathrm{~nm}$ band. Finally, the dye states relax to the lowest energy state on a nanosecond timescale. The final state that is occupied after several nanoseconds is the lowest energy state of the composite system. It is localized on the dye and emits around $700 \mathrm{~nm}$.
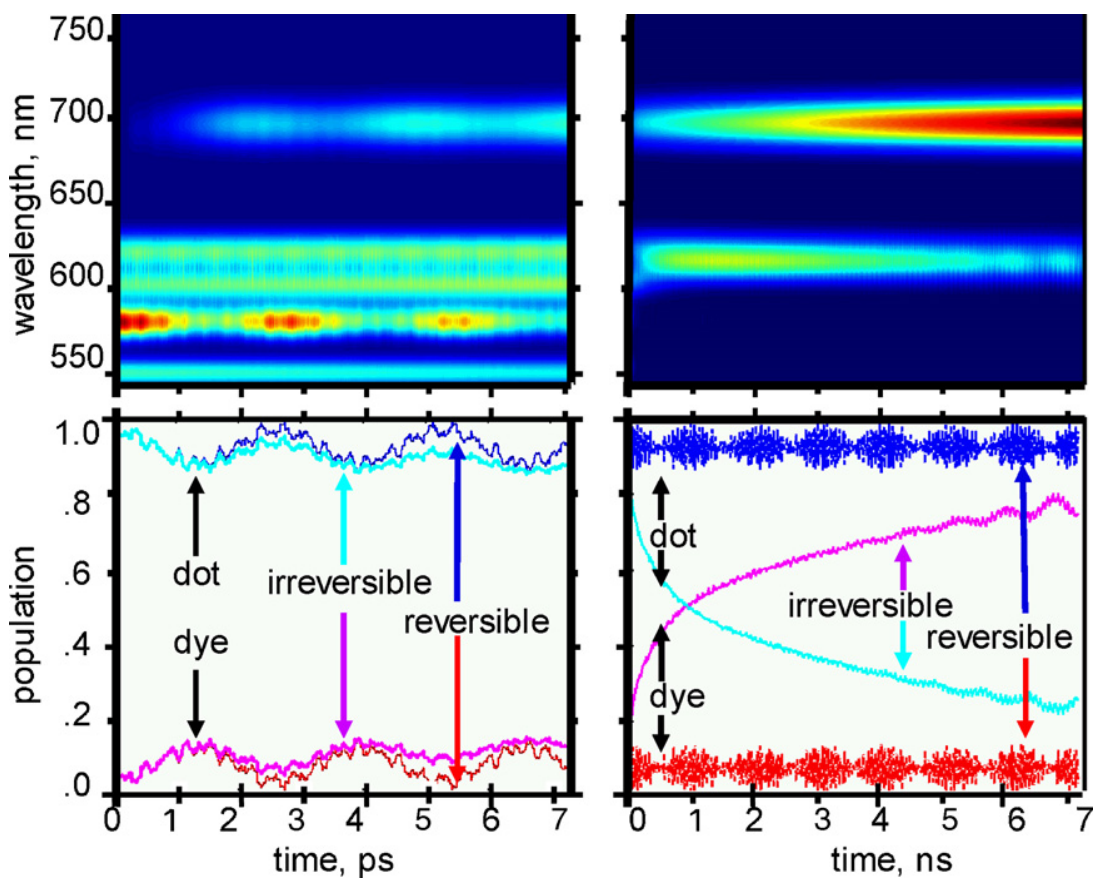

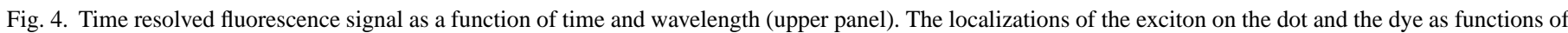

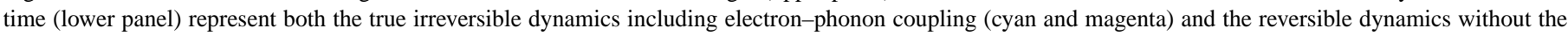
phonon bath (blue and red). 
In order to analyze the role that the phonon bath plays in the excitation transfer dynamics we consider the following two transfer regimes: First, we keep only the Liouville term in the EOM for the density matrix and neglect the interaction with phonons. This situation approximately corresponds to zero temperature. Second, we include the Redfield component into the EOM at ambient temperature. The two types of dynamics are illustrated in Fig. 4. The evolution of the exciton decoupled from the bath is reversible and shows pronounced beats with the period of $2.5 \mathrm{ps}$. The complete model including the interaction with the bath produces irreversible dynamics. The differences between the reversible and irreversible evolution appear after about $5 \mathrm{ps}$. The coupling to the phonon bath results in exponential decay of the population of the higher energy states and is responsible for the detailed balance between the transitions upward and downward in energy. While the overall irreversible transfer takes a few nanoseconds, each elementary act of transfer is much shorter. Thus, the overall transfer occurs by a sequence of small amplitude transfer events that are made irreversible by interaction with phonons. At the single molecule level, this average result can be expected to represent a broad distribution of individual transfer times. The fast oscillations in the spectral signal associated with the elementary transfer events can be observed by femtosecond-resolved spectroscopy.

The dynamics of the excitation transfer may be complicated by the presence of SOI. Experimental data indicates that the lowest energy exciton in $\mathrm{CdSe}$ QDs is a mixture of singlet and triplet states [81]. The sequence of events involving an optical excitation in the manifold of singlet states and a subsequent transfer of a singlet exciton can be enriched by intersystem crossing (ISC) and formation of a triplet exciton. The dipole-dipole coupling approximation applies directly only to singlet exciton transfer but by virtue of the ISC, it remains valid for the description of triplet states as well $[83,84,89]$. The time-resolved experimental data [81] indicates that ISC between the singlet and triplet state manifolds of QDs occurs faster than the excitation transfer between the dot and the dye [28]. Therefore, one can expect an average behavior that is well represented by the present model.

The reported simulations show that the photoinduced energy transfer is the preferred evolution pathway. The result agrees with the analysis of Clapp et al. [90]. In the experiments of Zenkevich et al. [28] the organic dye is put in direct contact with the QD. This arrangement may induce additional competing processes, including (i) charge transfer and (ii) relaxation to dark states, that reduce the efficiency of the energy transfer. Charge transfer followed by non-radiative return to the ground state can prevent the desired energy transfer from the QD to the dye. This pathway was not efficient in the current simulation, since the overall energy gradient towards the dye created favorable conditions for the transfer of energy rather than charge. If phonon-induced relaxation leads the system into a dark state, i.e. an excited state with a negligibly small transition dipole moment, both Förster energy transfer and fluorescence are impossible, and non-radiative relaxation becomes the most likely pathway. Dark states are not important in the present case, since the lowest energy excitons localized on both the dot and the dye are bright. The lowest energy bright state of the dot is efficiently transfered onto the dye by the Förster mechanism. The lowest energy bright state of the dye either emits, or transfers its energy to the oxygen, if the latter is present.

\section{Conclusions}

The photoexcitation transfer in a core-shell CdSe/ZnS QD sensitized with a meta-dipyridyl-free-base-porphyrin has been studied by $a b$ initio electronic structure and reduced density matrix equations-of-motion theories. The initial excitation is transferred from the dot to the dye in a resonance fashion and remains on the dye due to irreversible relaxation induced by a thermalized phonon bath. As a result, the emission of the quantum dot is quenched, giving rise to the emission of the dye. The excitation transfer occurs on a nanosecond time-scale, in agreement with the experiment [28].

The investigated photoinduced energy transfer holds great promise for applications in PDT, in which malignant tissues are destroyed by singlet oxygen. In order to circumvent the optical selection rule forbidding direct photoexcitation of the triplet ground state of oxygen, the $\mathrm{QD} /$ porphyrin assembly is designed to act as an active photodynamic agent capable of transferring the photon energy to the oxygen. Compared to simple dyes, the application of the QD/porphyrin assembly studied in the present work carries great advantages for PDT. The absorption spectra of porphyrins and phthalocyanines used in PDT show intense bands at the boundary between ultraviolet (UV) and visible light, known as Soret bands, and in the region between visible and near infrared light, known as Q-bands [91]. The extinction coefficients of the two types of bands have the same magnitude as those of QDs, on the order of $10^{5} \mathrm{M}^{-1} \mathrm{~cm}^{-1}$. The absorbance of the porphyrin and phthalocyanine dyes in the wide region between the Soret and bands is lower by several orders of magnitude. In contrast, the absorbance of QDs is high over the continuous region from the first excitonic band to UV. The $\mathrm{QD} /$ dye assemblies are much more efficient harvesters of light over a broad spectral region than individual dyes at the same concentration and excitation conditions. In order to reach an equivalent level of absorbance efficiency the concentration of individual dyes should be 1-3 orders of magnitude higher than the concentration of QD/dye complexes [91].

The reported simulations provide the first state-of-the-art $a b$ initio description of the electronic structure of the porphyrin/QD system and pioneer the application of the reduced density matrix theory to the long-time dynamics of the two-particle excitation transfer in a realistic dye/core-shell-QD assembly. The combination of the two approaches has allowed us to compute the experimentally studied time-resolved spectroscopic observables in a large, heavy-atom system over a long period of time. The simulations create the following scenario for the photoinduced excitation transfer in the dot-dye assembly: (i) the optical pulse generates an exciton by promoting an electron into the QD conduction band and creating a hole in the valence band, (ii) the exciton relaxes inside the dot over several picoseconds, (iii) the exciton resonantly transfers from the dot to the dye over hundreds of picoseconds, (iv) the transfered exciton relaxes inside the dye, (v) the electron and hole localized on the dye recombine 
by fluorescing at the emission frequency of the dye. If present, an oxygen molecule accepts the energy from dye, resulting in additional transfer steps.

The approach taken in this work can be further extended by explicit description of atomic vibrations and calculation of the electronic energies and dipole-dipole coupling for each nuclear configuration. As discussed above, a more rigorous model for the excited states can be used. Both improvements require significant computational cost. Explicit modeling of the subsequent excitation transfer from the porphyrin to the dioxygen will complete the theoretical description of the primary step in the PDT.

In addition to the energy transfer studied in this work, several alternative processes can occur in the porphyrin/QD aggregates and should be modeled in order to prove that they are not important. In particular one should carefully investigate the efficiency of the charge transfer, which may compete with the energy transfer and can be sensitive to the details of the geometric and electronic structure of the dye-dot assembly. The transfer of either hole or electron from the $\mathrm{CdSe}$ core to the dye or $\mathrm{ZnS}$ surface is undesired, since it will diminish the porphyrin fluorescence. The porphyrin tends to be a better electron-acceptor than hole-acceptor. Electron transfer from the dot to the dye is one of the main competing mechanisms. On the other hand, the hole can be trapped in a surface state of the QD. The efficiency of these processes can be most appropriately calculated by the nonadiabatic techniques. The relatively long distance between the dot and the porphyrin molecule disfavors the electron transfer. The hole trapping is prevented by the $\mathrm{ZnS}$ shell that saturates the dangling bonds of the CdSe core, eliminating the surface states. In addition, the $\mathrm{ZnS}$ shell provides a tunneling barrier for the electron transfer, since $\mathrm{ZnS}$ has a larger electron energy gap than both CdSe and the porphyrin. The good agreement between the experimentally determined and calculated changes in the optical signals supports the hypothesis that the energy transfer from the dot to the dye is indeed the major photoexcitation dynamics pathway, and is responsible for quenching the QD fluorescence. The detailed understanding of the mechanism of the photoreaction in the QD/dye aggregate provided by the reported simulations creates a clear picture of the process and will assist scientists in selecting types of QDs and photosensitizers that will optimize the production of singlet oxygen.

\section{Acknowledgments}

The authors are grateful to Martin Gouterman, Sergei Tretiak, and Svetlana Kilina for fruitful discussions and thank Colleen Craig for comments on the manuscript. The research was supported by the United States Department of Energy Grant no. DE-FG02-05ER15755 to O.V. P. and the INTAS Grant 03-504540 "Optical Active Assemblies of Colloidal Quantum Dots and Tetrapyrrolic Compounds" to E.I. Z. and C. v. B.

\section{References}

[1] M. Ouyang, D.D. Awschalom, Science 301 (2003) 1074.

[2] J. Gorman, D. Hasko, D. Williams, Phys. Rev. Lett. 95 (2005) 090502.
[3] J. Petta, A. Johnson, J. Taylor, E. Laird, A. Yacoby, M. Lukin, C. Marcus, M. Hanson, A. Gossard, Science 309 (2005) 2180.

[4] A.J. Nozik, Annu. Rev. Phys. Chem. 52 (2001) 193.

[5] J.E. Murphy, M.C. Beard, A.G. Norman, S.P. Ahrenkiel, J.C. Johnson, P. Yu, O.I. Micic, R.J. Ellingson, A.J. Nozik, J. Am. Chem. Soc. 128 (2006) 3241.

[6] R.D. Schaller, V.I. Klimov, Phys. Rev. Lett. 92 (2004) 186601.

[7] R.D. Schaller, V.M. Agranovich, V.I. Klimov, Nat. Phys. 1 (2005) 189.

[8] Q. Shen, D. Arae, T. Toyoda, J. Photochem. Photobiol. A: Chem. 164 (2004) 75 .

[9] S.H. Choi, H.J. Song, I.K. Park, J.H. Yum, S.S. Kim, S.H. Lee, Y.E. Sung, J. Photochem. Photobiol. A Chem. 179 (2006) 135.

[10] M. Neges, K. Schwartzburg, F. Willig, Sol. Energy Mater. Sol. Cells 90 (2006) 2107.

[11] R. Scheibner, H. Buhmann, D. Reuter, M. Kiselev, L. Molenkamp, Phys. Rev. Lett. 95 (2005) 176602.

[12] D. Talapin, C. Murray, Science 310 (2005) 86.

[13] S. Coe, W. Woo, M. Bawendi, V. Bulovic, Nature 420 (2002) 800.

[14] V.I. Klimov, A.A. Mikhailovsky, S. Xu, A. Malko, J.A. Hollingsworth, C.A. Leatherdale, H.J. Eisler, M.G. Bawendi, Science 290 (2000) 314.

[15] J. Farahani, D. Pohl, H. Eisler, B. Hecht, Phys. Rev. Lett. 95 (2005) 017402.

[16] E. Katz, I. Willner, Angew. Chem. Int. Ed. 43 (2004) 6042.

[17] M. Dahan, S. Levi, C. Luccardini, P. Rostaing, B. Riveau, A. Triller, Science 302 (2003) 442.

[18] J.M. Tsay, X. Michalet, Chem. Biol. 12 (2005) 1159.

[19] W.M. Sharman, C.M. Allen, J.E. van Lier, Drug Discovery Today 5 (1999) 507.

[20] T.J. Dougherty, J.G. Levy, in: T. Vo-Dinh (Ed.), Photodynamic Therapy (PDT) and Clinical Applications, CRC Press, Washington, DC, 2003 (Chapter 38).

[21] M. Ferrari, Curr. Opin. Chem. Biol. 9 (2005) 343.

[22] A.C.S. Samia, X.B. Chen, C. Burda, J. Am. Chem. Soc. 125 (2003) 15736.

[23] S. Lee, L. Zhu, A. Minhai, M.F. Hinds, A.A. Ferrante, D.H. Vu, D. Rosen, S.J. Davis, T. Hasan, Proc. SPIE Int. Soc. Opt. Eng. 5689 (2005) 90.

[24] R. Bakalova, H. Ohba, Z. Zhelev, M. Ishikawa, Y. Baba, Nat. Biotechnol. 22 (2004) 1360.

[25] A.P. Alivisatos, W.W. Gu, C. Larabell, Ann. Rev. Biomed. Eng. 7 (2005) 55.

[26] S. Dayal, R. Krolicki, Y. Lou, X. Qiu, J.C. Berlin, M.E. Kenney, C. Burda, Appl. Phys. B 84 (2006) 309.

[27] E.I. Zenkevich, E. Sagun, V. Knyukshto, A. Shulga, A. Mironov, O. Efremova, R. Bonnett, S.P. Songca, H. Kassem, J. Photochem. Photobiol. B: Biol. 33 (1996) 171.

[28] E.I. Zenkevich, F. Cichos, A. Shulga, E.P. Petrov, T. Blaudeck, C. von Bor czyskowski, J. Phys. Chem. B 109 (2005) 8679.

[29] E. Zenkevich, T. Blaudeck, M. Abdel-Mottaleb, F. Cichos, A. Shulga, C. von Borczyskowski, Int. J. Photoenergy 7 (2006) 90242.

[30] I.L. Medintz, H.T. Ueda, E.R. Coldman, H. Mattoussi, J. Am. Chem. Soc. 126 (2004) 30.

[31] Y.G. Sun, B. Mayers, Y.N. Xia, Adv. Mater. 15 (2003) 641.

[32] G.R. Fleming, G.D. Scholes, Nature 431 (2004) 256.

[33] B.R. Green, W.W. Parson, Light-Harvesting Antennas in Photosynthesis, Kluwer, Dordrecht, 2003.

[34] H. van Amerongen, L. Valkunas, R. van Grondelle, Photosynthetie Exeitons, World Scientific, New Jersey, 2000.

[35] Y.C. Cheng, R.J. Silbey, Phys. Rev. Lett. 96 (2006) 028103.

[36] V.I. Novoderezhkin, A.G. Yakovlev, R. van Grondelle, V.A. Shuvalov, J. Phys. Chem. B 108 (2004) 7445.

[37] E.I. Zenkevich, D.S. Kilin, A. Willert, S.M. Bachilo, A.M. Shulga, U. Remel, C. von Borczyskowski, Mol. Cryst. Liq. Cryst. 361 (2001) 83.

[38] E.I. Zenkevich, A. Willert, S.M. Bachilo, U. Rempel, D.S. Kilin, A.M. Shulga, C. von Borczyskowski, Mater. Sci. Eng. C 18 (2001) 99.

[39] M. Gruebele, J. Phys. Condens. Matter 16 (2004) R1057.

[40] J.A. Cina, G.R. Fleming, J. Phys. Chem. A 108 (2004) 11196.

[41] S. Mukamel, Ann. Rev. Phys. Chem. 51 (2000) 691.

[42] Q. Shi, E. Geva, J. Chem. Phys. 120 (2004) 10647.

[43] Q. Shi, E. Geva, J. Chem. Phys. 119 (2003) 11773.

[44] M. Schreiber, D. Kilin, U. Kleinekathöfer, J. Lumin. 83\&84 (1999) 235. 
[45] J.A. Cina, D.S. Kilin, T.S. Humble, J. Chem. Phys. 118 (2003) 46.

[46] T. Förster, in: O. Sinanoglu (Ed.), Modern Quantum Chemistry, Academic Press, New York, 1965, p. 93.

[47] M. Gruebele, P.G. Wolynes, Acc. Chem. Res. 37 (2004) 261.

[48] J.C. Tully, in: B.J. Berne, G. Cicotty, D.F. Coker (Eds.), Classical and Quantum Dynamics in Condenced Phase Simulations, World Scientific, New Jersey, 1998.

[49] H.B. Wang, M. Thoss, W.H. Miller, J. Chem. Phys. 115 (2001) 2979.

[50] O.V. Prezhdo, P.J. Rossky, J. Chem. Phys. 107 (1997) 825.

[51] O.V. Prezhdo, J. Chem. Phys. 111 (1999) 8366.

[52] O.V. Prezhdo, C. Brooksby, Phys. Rev. Lett. 86 (2001) 3215.

[53] C.F. Craig, W.R. Duncan, O.V. Prezhdo, Phys. Rev. Lett. 95 (2005) 163001

[54] O.V. Prezhdo, Theor. Chem. Acc. 116 (2006) 206.

[55] D.V. Matyushov, J. Chem. Phys. 120 (2004) 7532.

[56] D.V. Matyushov, J. Chem. Phys. 122 (2005) 044502.

[57] O.V. Prezhdo, P.J. Rossky, J. Phys. Chem. 100 (1996) 17094.

[58] A.A. Mosyak, O.V. Prezhdo, P.J. Rossky, J. Chem. Phys. 109 (1998) 6390.

[59] O.V. Prezhdo, V.V. Prezhdo, J. Mol. Struct. (Theochem.) 526 (2000) 115.

[60] C. Brooksby, O.V. Prezhdo, P.J. Reid, J. Chem. Phys. 118 (2003) 4563.

[61] C. Brooksby, O.V. Prezhdo, P.J. Reid, J. Chem. Phys. 119 (2003) 9111.

[62] S. Ramakrishna, F. Willig, V. May, Phys. Rev. B 62 (2000) R16330.

[63] L.X. Wang, F. Willig, V. May, J. Chem. Phys. 124 (2006) 014712.

[64] P.A. Apanasevich, S.Y. Kilin, A.P. Nizovtsev, N.S. Onishchenko, J. Opt. Soc. Am. B 3 (1986) 587.

[65] S. Yang, J. Cao, J. Chem. Phys. 121 (2002) 562.

[66] M. Schreiber, P. Herman, I. Barvik, I. Kondov, U. Kleinekathöfer, J. Lumin. 108 (2004) 137.

[67] O.V. Prezhdo, Phys. Rev. Lett. 85 (2000) 4413.

[68] D.S. Kilin, E.I. Zenkevich, C. von Borczyskowski, O.V. Prezhdo, Abstr. Papers Americal Chem. Soc. 230 (2005) U2971.

[69] A. Kasuya, R. Sivamohan, Y.A. Barnakov, I.M. Dmitruk, T. Nirasawa, V.R. Romanyuk, V. Kumar, S.V. Mamykin, K. Tohji, B. Jeyadevan, K.
Shinoda, T. Kudo, O. Terasaki, Z. Liu, R.V. Belosludov, V. Sundararajan, Y. Kawazoe, Nat. Mater. 3 (2004) 99.

[70] G. Kresse, J. Furtmüller, Phys. Rev. B 54 (1996) 11169.

[71] G. Kresse, J. Hafner, Phys. Rev. B 49 (1996) 14251.

[72] G. Kresse, J. Furtmüller, Comput. Mater. Sci. 6 (1996) 16.

[73] D. Vanderbilt, Phys. Rev. B 41 (1990) 7892.

[74] J.P. Perdew, Y. Wang, Accurate and simple analytic representation of the electron-gas correlation energy, Phys. Rev. B 45 (1992) 13244-13249.

[75] HyperChem (TM), Lite v. 2 (Hypercube, Inc., 1115 NW 4th Street, Gainesville, Florida 32601, USA, 2000).

[76] O.A.V. Lilienfeld, I. Tavernelli, U. Rothlisberger, D. Sebastiani, Phys. Rev. B 71 (2005) 195119.

[77] Y. Zhao, D.G. Truhlar, Phys. Chem. Chem. Phys. 7 (2005).

[78] B.I. Stepanov, Nature 157 (1946) 808.

[79] M. Rohlfing, S.G. Louie, Phys. Rev. B 62 (2000) 4927.

[80] E.J. Bylaska, K. Tsemekhman, F. Gao, Phys. Scr. T 124 (2006) 86.

[81] G.D. Scholes, J. Kim, C.Y. Wong, V.M. Huxter, P.S. Nair, K.P. Fritz, S. Kumar, Nano Lett. 6 (2006) 1765.

[82] F.W. Wise, Acc. Chem. Res. 33 (2000) 773

[83] G.P. Gurinovich, E.I. Zenkevich, E.I. Sagun, J. Lumin. 26 (1982) 297.

[84] M.A. El-Sayed, Acc. Chem. Res. 1 (1968) 8.

[85] A. Dreuw, M. Head-Gordon, J. Am. Chem. Soc. 126 (2004) 4007.

[86] O.V. Prezhdo, Adv. Mater. 14 (2002) 592.

[87] A.L. Efros, M. Rosen, M. Kuno, M. Nirmal, D.J. Norris, M. Bawendi, Phys. Rev. B 54 (1996) 4843.

[88] M. Gouterman, J. Mol. Spectrosc. 6 (1961) 138.

[89] D. Gust, T.A. Moore, A.L. Moore, C. Devadoss, P.A. Liddell, R. Hermant, R.A. Nieman, L.J. Demanche, J.M. DeGraziano, I. Gouni, J. Am. Chem. Soc. 114 (1992) 3590.

[90] A.R. Clapp, I.L. Medintz, J.M. Mauro, B.R. Fisher, M.G. Bawendi, H. Mattoussi, J. Am. Chem. Soc. 126 (2004) 301.

[91] A.C.S. Samia, S. Dayal, C. Burda, Photochem. Photobiol. 82 (2006) 617. 